\title{
Variation in the autism candidate gene GABRB3 modulates tactile sensitivity in typically developing children
}

\author{
Teresa Tavassoli ${ }^{1}{ }^{2 *}$, Bonnie Auyeung ${ }^{1}$, Laura C Murphy ${ }^{1}$, Simon Baron-Cohen ${ }^{1}$ and Bhismadev Chakrabarti ${ }^{1,3}$
}

\begin{abstract}
Background: Autism spectrum conditions have a strong genetic component. Atypical sensory sensitivities are one of the core but neglected features of autism spectrum conditions. GABRB3 is a well-characterised candidate gene for autism spectrum conditions. In mice, heterozygous Gabrb3 deletion is associated with increased tactile sensitivity. However, no study has examined if tactile sensitivity is associated with GABRB3 genetic variation in humans. To test this, we conducted two pilot genetic association studies in the general population, analysing two phenotypic measures of tactile sensitivity (a parent-report and a behavioural measure) for association with 43 SNPS in GABRB3.

Findings: Across both tactile sensitivity measures, three SNPs (rs11636966, rs8023959 and rs2162241) were nominally associated with both phenotypes, providing a measure of internal validation. Parent-report scores were nominally associated with six SNPs $(P<0.05)$. Behaviourally measured tactile sensitivity was nominally associated with 10 SNPs (three after Bonferroni correction).

Conclusions: This is the first human study to show an association between GABRB3 variation and tactile sensitivity. This provides support for the evidence from animal models implicating the role of GABRB3 variation in the atypical sensory sensitivity in autism spectrum conditions. Future research is underway to directly test this association in cases of autism spectrum conditions.
\end{abstract}

Keywords: Autism spectrum conditions, autistic traits, GABA, sensory profile, tactile sensitivity

\section{Findings \\ Background}

Twin studies of autism spectrum conditions (ASC) and autistic traits show high heritability, suggesting a strong genetic component $[1,2]$. In addition to social and communication deficits, individuals with ASC often show atypical tactile sensitivity, for example, not wanting to wear certain items of clothing [3-5]. However, little is known about mechanisms underlying normative variability in tactile sensitivity. Specifically, the relationship between genotypes and sensory phenotypes is largely unknown. A large scale heritability study on approximately 1,400 twins suggested a moderate genetic influence on

\footnotetext{
* Correspondence: teresa.tavassoli@mssm.edu

'Department of Psychiatry, Autism Research Centre, University of Cambridge, Cambridge, UK

${ }^{2}$ Current address: Seaver Autism Center, Mount Sinai, NY, USA

Full list of author information is available at the end of the article
}

tactile sensitivity using parent reports, showing a heritability estimate of $52 \%$ [6].

Multiple lines of evidence suggest that the normative variability in tactile sensitivity is related to differences in gamma-aminobutyric acid (GABA) function. For example, heterozygous Gabrb3 deletion causes increased tactile sensitivity (that is, hypersensitivity) in male mice [7]. In humans, a Magnetic Resonance Spectroscopy study reported that tactile sensitivity, or narrower tactile discrimination thresholds, are significantly associated with GABA levels in the sensorimotor cortex [8]. GABA is a neurotransmitter that is crucial in early cortical development as it has an important role in both excitatory and inhibitory synapses $[9,10]$. Later in development, GABA is the main inhibitory transmitter in the brain. GABRB3 codes for one of the principal GABA receptors in the human brain, and is one of the most validated candidate genes for ASC in both humans as well as in

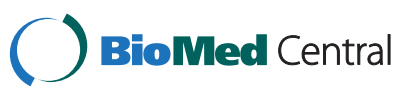


animal models [11-13]. Genetic variations within the GABAergic system have been repeatedly associated with ASC and autism-related traits [11-16], though with some non-replications [17]. In view of the strong evidence suggesting an association of GABRB3 variation with ASC, and specifically a role of Gabrb3 in tactile sensitivity in mice, we aimed to test variation in this gene for its role in tactile sensitivity in humans.

The current studies report a preliminary investigation of underlying genetic mechanisms of tactile sensitivity in typically developing children. The aim was to test an association between variation in GABRB3 and two tactile sensitivity phenotypes (measured through a parentreport and a behavioural touch test). The experiment was designed to ensure that the behavioural touch test was identical to that used in mice by DeLorey et al. [7] (using the Semmes Weinstein Von Frey Aesthesiometer for touch assessment test). We hypothesized an association between variations in GABRB3 and tactile sensitivity measured using the touch test. We also hypothesized an association between GABRB3 variation and tactile sensitivity measured using a tactile subscale of a parentreport, the Short Sensory Profile (SSP). The SSP has been used in ASC, demonstrating differences in tactile sensitivity [18].

To our knowledge, no previous study has investigated if commonly occurring genetic variations have an impact on normative variation in human tactile sensitivity. Understanding sensory perceptual variation in the general population could guide the study of genetic mechanisms underlying sensory issues in ASC.

\section{Methods}

This study was given ethical approval by the National Health Service Suffolk Research Ethics Committee (Reference: 05/Q0102/16).

\section{Participants}

Signed written consent forms were obtained from 87 parents who then filled in the SSP for their child. Scores were calculated for the SSP tactile subscale ( 44 boys, 43 girls; children's mean age: 9.00 years \pm 3.22 years). A subset of 39 children took part in the behavioural measured tactile sensitivity study ( 21 boys, 18 girls; mean age: 11.51 years \pm 0.87 years). The Wechsler Abbreviated Scale of Intelligence was used to measure the intelligence quotient (IQ) (mean Full Scale IQ: $117.12 \pm 14.38)$.

\section{Short sensory profile (parent-report measure)}

The SSP is a caregiver questionnaire with 38 items investigating daily life sensory experiences in children (http://www.pearsonassessments.com). For the current study, the SSP tactile subscale score including seven items was calculated [19]. Internal reliability of the tactile sensitivity subscale ranges from 0.70 to 0.84 [19]. Parents were asked to describe the frequency ( 1 = always; 5 $=$ never) with which their child performed behaviours such as 'avoids going barefoot, especially in sand or grass (item 3)'.

\section{Touch test (behavioural measure)}

Tactile sensitivity was measured using the Semmes Weinstein Von Frey Aesthesiometer for Touch Assessment test (Stoelting Co., Wood Dale, IL, USA), with a forced-choice method of limits paradigm (left or right). Each filament is individually calibrated to deliver its target force within a $5 \%$ standard deviation. The participant's arms were at rest. The experimenter lowered the filaments manually until skin contact was made for 1.5 seconds. The participant was asked to close their eyes throughout. After this, the participant reported where they detected the stimulus by saying 'left' or 'right'. The order was randomized. A 'method of limits' procedure with three descending and three ascending trials was applied. The first descending trial started with a thick filament that all participants were able to detect (4.31 g force). Then the next thinnest filament was applied until the participant could no longer detect the stimulus for two consecutive trials. The ascending trial started by increasing the force until the participant could detect the filament in two consecutive trials.

\section{Single nucleotide polymorphism selection}

Multiple SNPs were selected using the following criteria: minor allele frequency $>0.1$ in a Caucasian population, and ensuring the maximum gene coverage through linkage disequilibrium [20]. A total of 43 SNPs were chosen, to provide maximal coverage of the gene. DNA was extracted from mouth swabs, which were then anonymized and genotyped using standard PCR-based assays (TaqMan SNP genotyping assays; Applied Biosystems Inc., Foster City, CA, USA). The genotyping call rate was $99.45 \%$. No SNP deviated significantly from HardyWeinberg equilibrium $(P<0.05)$ (as determined using http://www.changbioscience.com/genetics/hardy).

\section{Statistical analysis}

UNPHASED 3.1.4 was used to test for genetic association. The following phenotypes were tested for association with all genotyped SNPs (see Additional file 1: Table S1 for all SNPs): parent-reported tactile sensitivity on the SSP, and behaviourally measured tactile sensitivity using a touch test. Bonferroni corrections were used to control for multiple comparisons (i.e. significance level was set to 0.05 and divided by the number of effective comparisons). The effective number of independent genotyped SNPs in GABRB3 (after accounting for linkage 
Table 1 List of all significant SNPs and their genotypic associations with tactile sensitivity as measured using the parent-report Short Sensory Profile and a behavioural touch test

\begin{tabular}{|c|c|c|c|c|c|c|c|c|}
\hline \multirow[t]{2}{*}{ Gene variations } & \multicolumn{4}{|c|}{ SSP tactile score (raw score) } & \multicolumn{4}{|c|}{ Behavioural touch test (g) } \\
\hline & mean & sd & $\mathrm{X}^{2}$ statistic & $P$ & mean & sd & $\mathrm{X}^{2}$ statistic & $P$ \\
\hline rs11636966 & & & 10.77 & $0.004^{*}$ & & & 10.18 & $0.006^{*}$ \\
\hline$C / C$ & 33.34 & 2.10 & & & 0.145 & 0.187 & & \\
\hline $\mathrm{C} / \mathrm{T}$ & 31.25 & 3.47 & & & 0.022 & 0.024 & & \\
\hline $\mathrm{T} / \mathrm{T}$ & 33.00 & 2.12 & & & 0.024 & 0.021 & & \\
\hline rs8023959 & & & 4.32 & $0.03^{*}$ & & & 4.70 & $0.02^{*}$ \\
\hline$A / A$ & 32.08 & 3.06 & & & 0.056 & 0.125 & & \\
\hline $\mathrm{A} / \mathrm{C}$ & 33.56 & 1.75 & & & 0.117 & 0.133 & & \\
\hline rs2162241 & & & 5.68 & $0.05^{*}$ & & & 7.07 & $0.02^{*}$ \\
\hline $\mathrm{C} / \mathrm{C}$ & 31.53 & 3.60 & & & 0.021 & 0.019 & & \\
\hline$C / \mathrm{T}$ & 32.95 & 2.20 & & & 0.087 & 0.123 & & \\
\hline $\mathrm{T} / \mathrm{T}$ & 33.12 & 1.64 & & & 0.141 & 0.258 & & \\
\hline rs7179514 & & & 10.82 & $0.004^{*}$ & & & 4.69 & 0.09 \\
\hline $\mathrm{G} / \mathrm{G}$ & 32.44 & 2.97 & & & 0.023 & 0.022 & & \\
\hline $\mathrm{G} / \mathrm{C}$ & 31.72 & 3.06 & & & 0.057 & 0.138 & & \\
\hline$C / C$ & 34.00 & 1.78 & & & 0.115 & 0.143 & & \\
\hline rs17117279 & & & 7.75 & $0.02^{*}$ & & & 1.72 & 0.42 \\
\hline $\mathrm{A} / \mathrm{A}$ & 32.69 & 2.80 & & & 0.009 & 0.136 & & \\
\hline $\mathrm{A} / \mathrm{T}$ & 31.96 & 2.64 & & & 0.060 & 0.115 & & \\
\hline $\mathrm{T} / \mathrm{T}$ & 23.00 & 0.00 & & & 0.080 & 0.000 & & \\
\hline rs7171512 & & & 5.72 & $0.05^{*}$ & & & 0.91 & 0.63 \\
\hline $\mathrm{G} / \mathrm{G}$ & 32.89 & 2.26 & & & 0.096 & 0.164 & & \\
\hline $\mathrm{G} / \mathrm{A}$ & 31.56 & 3.44 & & & 0.050 & 0.096 & & \\
\hline$A / A$ & 33.20 & 2.31 & & & 0.027 & 0.028 & & \\
\hline rs737098 & & & 2.73 & 0.25 & & & 17.75 & $0.0001^{* *}$ \\
\hline $\mathrm{A} / \mathrm{A}$ & 31.96 & 3.46 & & & 0.015 & 0.012 & & \\
\hline$A / G$ & 32.92 & 2.03 & & & 0.129 & 0.172 & & \\
\hline $\mathrm{G} / \mathrm{G}$ & 32.60 & 1.52 & & & 0.033 & 0.033 & & \\
\hline rs1367959 & & & 4.43 & 0.10 & & & 13.13 & $0.001^{*}$ \\
\hline$C / C$ & 32.62 & 3.03 & & & 0.089 & 0.143 & & \\
\hline $\mathrm{C} / \mathrm{T}$ & 31.65 & 2.79 & & & 0.010 & 0.005 & & \\
\hline $\mathrm{T} / \mathrm{T}$ & 33.55 & 2.38 & & & 0.019 & 0.015 & & \\
\hline rs1426224 & & & 0.13 & 0.71 & & & 9.78 & $0.001^{*}$ \\
\hline $\mathrm{T} / \mathrm{T}$ & 32.30 & 3.00 & & & 0.040 & 0.074 & & \\
\hline T/C & 32.67 & 2.06 & & & 0.215 & 0.235 & & \\
\hline rs3212331 & & & 3.40 & 0.18 & & & 11.68 & $0.002^{*}$ \\
\hline$A / A$ & 32.04 & 3.19 & & & 0.078 & 0.108 & & \\
\hline$A / G$ & 32.56 & 2.60 & & & 0.055 & 0.164 & & \\
\hline $\mathrm{G} / \mathrm{G}$ & 33.86 & 1.86 & & & 0.008 & 0.000 & & \\
\hline rs8026392 & & & 1.42 & 0.48 & & & 11.71 & $0.002^{*}$ \\
\hline $\mathrm{T} / \mathrm{T}$ & 32.05 & 3.26 & & & 0.019 & 0.019 & & \\
\hline $\mathrm{T} / \mathrm{C}$ & 32.62 & 2.76 & & & 0.120 & 0.171 & & \\
\hline $\mathrm{C} / \mathrm{C}$ & 33.13 & 1.46 & & & 0.029 & 0.026 & & \\
\hline
\end{tabular}


Table 1 List of all significant SNPs and their genotypic associations with tactile sensitivity as measured using the parent-report Short Sensory Profile and a behavioural touch test (Continued)

\begin{tabular}{|c|c|c|c|c|c|c|c|c|}
\hline rs11161329 & & & 3.02 & 0.21 & & & 7.29 & $0.02^{*}$ \\
\hline$A / A$ & 32.49 & 2.93 & & & 0.053 & 0.122 & & \\
\hline$A / T$ & 31.89 & 3.00 & & & 0.032 & 0.039 & & \\
\hline $\mathrm{T} / \mathrm{T}$ & 33.29 & 2.76 & & & 0.138 & 0.189 & & \\
\hline rs12905535 & & & 3.88 & 0.14 & & & 7.42 & $0.02^{*}$ \\
\hline $\mathrm{T} / \mathrm{T}$ & 31.74 & 3.55 & & & 0.019 & 0.019 & & \\
\hline $\mathrm{T} / \mathrm{C}$ & 32.95 & 2.00 & & & 0.113 & 0.169 & & \\
\hline $\mathrm{C} / \mathrm{C}$ & 32.60 & 1.52 & & & 0.033 & 0.033 & & \\
\hline
\end{tabular}

${ }^{*} P \leq 0.05 .{ }^{* * P} \leq 0.001$. sd: standard deviation.

disequilibrium in this sample) is approximately 30, according to the Li and Ji correction [21]. The Bonferroni corrected $P$-value was thus set to $0.001(<0.05 / 30)$.

\section{Results}

\section{Descriptive statistics}

The parent-report SSP tactile score $(32.36 \pm 2.80)$ was in the average range (typical performance for SSP tactile: 35 to 30). High scores on the SSP represent typical performance [19]. Behavioural tactile sensitivity was also in the normal range (mean: $0.06 \mathrm{~g} \pm 0.12 \mathrm{~g}$ ) [22]. The higher the tactile sensitivity the less sensitive the participant is to touch.

\section{Association between GABRB3 and tactile sensitivity}

Genotypic association (2 degrees of freedom) analysis was conducted using UNPHASED. Both tactile sensitivity measures, that is, the parent-reported SSP tactile score and the behavioural touch test, showed a nominally significant association with three SNPs (rs11636966, rs8023959 and rs2162241) (see Table 1 and Figure 1).

Ten SNPs were nominally associated at $P<0.05$ with behaviourally measured tactile sensitivity (see Table 1 ).

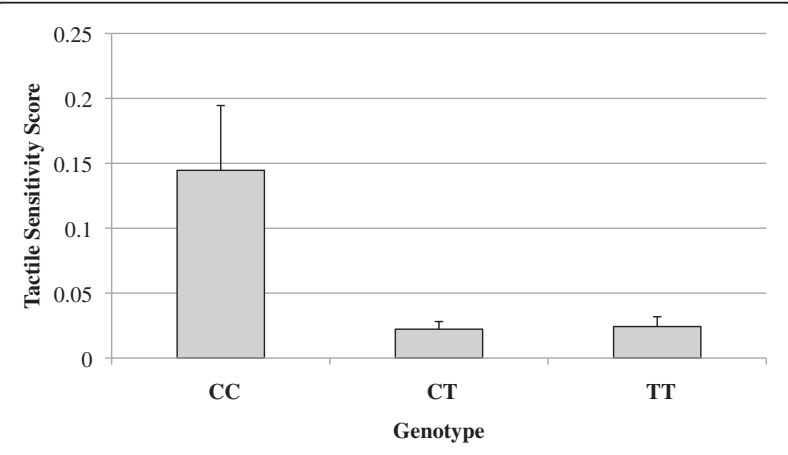

Figure 1 Association between behaviourally measured tactile sensitivity and rs11636966, a single nucleotide polymorphism in the GABRB3 gene. Tactile sensitivity thresholds $(\mathrm{g})$ varied as a function of genotype in this polymorphism. Error bars depict 1 standard error of mean.
After Bonferroni correction for the effective total number of SNPs [21], three SNPs were found to be significant: rs737098, rs1367959 and rs1426224 were associated with tactile sensitivity at $P<0.001$. Six SNPs were nominally associated (at $P<0.05$ ) with the parent-report SSP tactile sensitivity, none of which survived Bonferroni correction.

\section{Discussion}

This is the first study to test for genetic variation underlying the normative variation in tactile sensitivity in humans. We found that three SNPs in GABRB3 (rs11636966, rs8023959 and rs2162241) were nominally associated with two measures of tactile sensitivity (a parent-report - the SSP - and a behavioural touch test) suggesting a degree of internal replication. We also found a nominal association between six GABRB3 SNPs and parent-reported tactile sensitivity on the SSP. Lastly, nominally significant associations between $10 \mathrm{SNPs}$ in the GABRB3 gene with behaviourally measured tactile sensitivity thresholds were found. After correcting for multiple comparisons, three of these associations remained significant (rs737098, rs1367959 and rs1426224).

These results show that, depending on the specific genotype in certain polymorphisms in the GABRB3 gene, an individual can have higher or lower tactile sensitivity, measured both through parent-report or a behavioural touch test. This finding is especially interesting because we expected an association based on the study by DeLorey et al. [7], which reported that heterozygous knock-out mice for Gabrb3 have higher tactile sensitivity, that is, they are more sensitive towards touch. In the current study, we used the same behavioural touch test to measure tactile sensitivity and found an association at $P<0.001$ with three SNPs: rs737098, rs1367959 and rs1426224. All of these are intronic in nature.

The observed effects therefore might be mediated through the following mechanisms operating individually or in combination: one or more of the intronic SNPs are in linkage disequilibrium with exonic variation that 
has a direct effect on gene transcription, and the intronic SNPs themselves might subtly alter the binding affinities for the transcription machinery, thus having an indirect impact on mRNA abundance, structure and/or stability. The exact functional role of these SNPs is however unknown, and can be determined only by directly studying the impact of the different alleles on the expression of the GABRB3 gene. If the relationship between GABRB3 levels and tactile sensitivity is equivalent in mice and humans, we can expect that the genotypes associated with high tactile sensitivity would also be associated with reduced GABRB3 expression. This needs to be tested directly through cell culture studies.

One limitation of the current study is the small sample size, especially for the behavioural touch test. However, we found three SNPs were nominally associated with both tactile sensitivity measures. Furthermore, even though the sample size was particularly small for the behavioural touch test $(n=39)$, we identified three significant associations after Bonferroni correction. The current studies are nonetheless preliminary in nature, and further data is needed using larger sample sizes.

Another limitation is the lack of an independent replication sample, which is partly due to tactile sensitivity not being a widely used phenotypic measure. Investigations in our laboratory are underway to replicate our findings in a larger sample size. Another limitation of the current work is that we only included typically developing children. It will be important to investigate implications for individuals with ASC in future studies. Our ongoing investigations also include children with ASC.

Little is known about the role of GABA-related effects on sensory perception, and despite its limitations the current study is an important first step. Only one mouse model has investigated the association between Gabrb3 and tactile sensitivity [7]. These are the first studies looking at an association in humans with relevant phenotypes, which will hopefully stimulate further research in the field. To fully understand underlying causes of atypical sensory sensitivity in ASC, multiple approaches are necessary [1]. Additionally, testing the interplay between environmental and biological influences on sensory sensitivity will be important for future work. The current investigation of the underlying genetic architecture of individual differences in tactile sensitivity opens up the possibility of disentangling the relative contribution of specific genes in different aspects of autistic symptoms.

\section{Additional file}

Additional file 1: Table S1. List of all SNPS genotyped and their genotypic associations with tactile sensitivity as measured using the parent-report Short Sensory Profile and a behavioural touch test. * denotes significant level of $P \leq 0.05$.

\section{Abbreviations}

ASC: autism spectrum conditions; GABA: gamma-aminobutyric acid; PCR: polymerase chain reaction; SNP: single nucleotide polymorphism; SSP: Short Sensory Profile.

\section{Competing interests}

The authors declare that they have no competing interests.

\section{Authors' contributions}

$\Pi T, B A, S B C$ and $B C$ designed the study. BA and $T$ collected the data. $L M, T T$ and $B C$ carried out the data analyses. All authors were involved in writing the manuscript and approved the final version.

\section{Acknowledgments}

TT was supported by Autistica and the Pinsent Darwin Trust during the period of this work. SBC and BC were supported by the MRC UK. SBC was also supported by the Wellcome Trust. This work was conducted in association with the NIHR CLAHRC for Cambridgeshire and Peterborough NHS Foundation Trust, the Nancy Lurie Marks Family Foundation, and Target Autism Genome. We are grateful to the participants for their generous cooperation and to Allen Chan, Vanessa Puetz, Stephanie Suessenbacher and Dr Carrie Allison for their help. Genotyping took place at the Wellcome Trust Sanger Institute. We thank the late Professor Leena Peltonen there for making this collaboration possible.

\section{Author details}

'Department of Psychiatry, Autism Research Centre, University of Cambridge, Cambridge, UK. ${ }^{2}$ Current address: Seaver Autism Center, Mount Sinai, NY, USA. ${ }^{3}$ Centre for Integrative Neuroscience and Neurodynamics, School of Psychology and Clinical Language Sciences, University of Reading, Reading, UK.

Received: 17 April 2012 Accepted: 8 June 2012 Published: 6 July 2012

\section{References}

1. Abrahams BS, Geschwind DH: Advances in autism genetics: on the threshold of a new neurobiology. Nat Rev Genet 2008, 9(5):341-355.

2. Bailey A, Le Couteur A, Gottesman I, Bolton P, Simmonoff E, Yuzda E, Rutter M: Autism as a strongly genetic disorder: evidence from a British twin study. Psychol Med 1995, 25:63-77.

3. Blakemore S-J, Tavassoli T, Calò S, Thomas RM, Catmur C, Frith U, Haggard P: Tactile sensitivity in Asperger syndrome. Brain Cogn 2006, 61(1):5-13.

4. Tommerdahl M, Tannan V, Cascio CJ, Baranek GT, Whitsel BL: Vibrotactile adaptation fails to enhance spatial localization in adults with autism. Brain Res 2007, 1154:116-123.

5. Cascio C, McGlone F, Folger S, Tannan V, Baranek G, Pelphrey KA, Essick G: Tactile perception in adults with autism: a multidimensional psychophysical study. J Autism Dev Disord 2008, 38(1):127-137.

6. Goldsmith HH, van Hulle CA, Arneson CL, Schreiber JE, Gernsbacher MA: A population-based twin study of parentally reported tactile and auditory defensiveness in young children. J Abnorm Child Psychol 2006, 34(3):393-407.

7. DeLorey TM, Sahbaie P, Hashemi, Li W, Salehi A, Clark D: Somatosensory and sensorimotor consequences associated with the heterozygous disruption of the autism candidate gene, Gabrb3. Behav Brain Research 2011, 216(1):36-45.

8. Puts NAJ, Edden RAE, Evans CJ, McClone F, McGonigle DJ: Regionally specific human GABA concentration correlates with tactile discrimination thresholds. J Neurosci 2011, 31:16556-16560.

9. Schmitz C, van Kooten IA, Hof PR, van Engeland H, Patterson PH, Steinbusch HW: Autism: neuropathology, alterations of the GABAergic system, and animal models. Int Rev Neurobiol 2005, 71:1-26.

10. DiCristo G: Development of GABAergic circuits and its implications for neurodevelopmental disorders. Clin Genet 2007, 72(1):1-8.

11. Buxbaum JD, Silverman JM, Smith CJ, Greenberg DA, Kilifarski M, Reichert J, Cook EH, Fang Y, Song CY, Vitale R: Association between a GABRB3 polymorphism and autism. Mol Psychiatry 2002, 7(3):311-316.

12. Pizzarelli R, Cherubini E: Alterations of GABAergic signaling in autism spectrum disorders. Neural Plast 2011, 2011:297153. 
13. Schroer RJ, Phelan MC, Michaelis RC, Crawford EC, Skinner SA, Cuccaro M, Simensen RJ, Bishop J, Skinner C, Fender D, Stevenson RE: Autism and maternally derived aberrations of chromosome 15q. Am J Med Genet 1998, 76(4):327-336.

14. Fatemi SH, Folsom TD, Reutiman TJ, Thuras PD: Expression of GABA(B) receptors is altered in brains of subjects with autism. Cerebellum 2009, 8(1):64-69.

15. Fatemi $S H$, Folsom TD, Thuras PD: Deficits in $G A B A(B)$ receptor system in schizophrenia and mood disorders: a postmortem study. Schizophr Research 2011, 128:37-43.

16. Chakrabarti B, Dudbridge F, Kent L, Wheelwright S, H-C G, Allison C, Banerjee-Basu S, Baron-Cohen S: Genes related to sex steroids, neural growth, and social-emotional behavior are associated with autistic traits, empathy, and Asperger syndrome. Autism Res 2009, 2(3):157-177.

17. Salmon B, Hallmayer J, Rogers T, Kalaydjieva L, Petersen PB, Nicholas P, Pingree C, McMahon W, Spiker D, Lotspeich L, Kraemer H, McCague P, Dimiceli S, Nouri N, Pitts T, Yang J, Hinds D, Myers RM, Risch N: Absence of linkage and linkage disequilibrium to chromosome 15q11-q13 markers in 139 multiplex families with autism. Am J Med Genet 1999, 88(5):551-556.

18. Tomchek SD, Dunn W: Sensory processing in children with and without autism: a comparative study using the short sensory profile. Am J Occup Ther 2007, 61(2):190-200.

19. Dunn W: The Sensory Profile Manual. San Antonio: The Psychological Corporation; 1999.

20. Collins FS, Guyer MS, Chakravarti A: Variations on a theme: cataloging human DNA sequence variation. Science 1997, 278:1580-1581.

21. Li J, Ji L: Adjusting multiple testing in multilocus analyses using the eigenvalues of a correlation matrix. Heredity 2005, 95:221-227.

22. Operational Manual: Touch Test Sensory Evaluators: Semmes Weinstein von Frey Hair Aesthesiometers. Wood Dale, LL: Stoelting Co; 2001. http://www. stoeltingco.com/stoelting/stores/stoelting/products/docs/ 1793_58011_touch_test_evaluator.pdf.

doi:10.1186/2040-2392-3-6

Cite this article as: Tavassoli et al:: Variation in the autism candidate gene GABRB3 modulates tactile sensitivity in typically developing children. Molecular Autism 2012 3:6.

\section{Submit your next manuscript to BioMed Central and take full advantage of:}

- Convenient online submission

- Thorough peer review

- No space constraints or color figure charges

- Immediate publication on acceptance

- Inclusion in PubMed, CAS, Scopus and Google Scholar

- Research which is freely available for redistribution 